\title{
Red de sensores inalámbrica para la detección de incendios forestales
}

\author{
Jorge Antonio Atempa Camacho, Arnoldo Díaz-Ramírez, \\ Rafael Iván Ayala Figueroa, Verónica Quintero Rosas \\ Instituto Tecnológico de Mexicali, Departamento de Sistemas y Computación, \\ México \\ \{jatempa, adiaz, rafaelivan, veronicaquintero\}@itmexicali.edu.mx
}

\begin{abstract}
Resumen. El problema de los incendios forestales es una amenaza constante para los ecosistemas del planeta. Su incidencia aumenta durante el verano, destruye su alrededor y provoca costos económicos incalculables en la recuperación de zonas boscosas, entre otros. Una red de sensores inalámbrica es una tecnología emergente con la capacidad de extraer datos de las condiciones ambientales del entorno para su procesamiento. La fusión de información permite combinar diferentes fuentes de datos para mejorar la calidad de la respuesta ante un evento de interés. En este artículo se propone un método para la detección de incendios forestales, utilizando una red de sensores inalámbrica y métodos de fusión de información. Su principal contribución es la implementación de un algoritmo de baja complejidad computacional con la capacidad de detectar un evento de incendio utilizando tan solo sensores de temperatura y humedad. La evaluación del método propuesto muestra una tasa de detección de incendios del $100 \%$ cuando los nodos de la red no están expuestos directamente a los rayos del sol.
\end{abstract}

Palabras clave: Wireless, sensores, redes, inalámbricas, WSN.

\section{Wireless Network of Sensors for Detection of Forest Fires}

\begin{abstract}
Forest fires represent a constant threaten to the world's ecosystems. They tend to occur more often in the summer season, destroying forest and urban areas, and causing incalculable economic and ecological damages. Wireless sensor networks are an emerging technology that is used to sense the environmental conditions of a predefined area, and to process the gathered data to detect events of interest. In addition, information fusion techniques use the data col- lected by different sources (i.e., sensors) and combine them to improve the human or automated decision-making process. In this paper, an early forest fire detection method is proposed, which is based on the use of a wireless sensor network and information fusion techniques. The main contribution of the proposed method is that it has a low computational complexity, which is appropriate for implement- ing real-time detection and notification systems. Also, it is a low-cost method since it uses only two sensors: temperature and relative humidity. The results of the evaluation of the proposed method showed
\end{abstract}


Jorge Antonio Atempa Camacho, Arnoldo Díaz-Ramírez, Rafael Iván Ayala Figueroa, et al.

a detection rate of $100 \%$ when the nodes of the network are not directly exposed to sunlight.

Keywords: Wireless, sensor, networks, WSN.

\section{Introducción}

Año con año México se ve afectado por el problema de los incendios forestales. Éstos representan una de las principales amenazas a los ecosistemas, ocasionando problemas tales como la erosión del suelo, la contaminación en el aire, la desaparición de especies nativas, la pérdida de vidas humanas y cuantiosos costos económicos para la recuperación de zonas boscosas [1]. De acuerdo con la Comisión Nacional Forestal (CONAFOR) de México, en su reporte anual de 2014, el estado de Baja California se ubicó en tercer lugar a nivel nacional al registrar 102 incendios forestales, los cuales dejaron 15,638.95 hectáreas de superficie afectada [2]. Éste es un problema que afecta a todo el planeta y preocupa a la comunidad científica.

Algunos métodos para la detección de incendios incluyen monitorización desde torres de vigilancia y el uso de imágenes satelitales [3, 4]. Desafortunadamente, estos no son eficientes debido a varias razones, tales como los costos elevados en infraestructura (equipo sofisticado), el que requieran un gran número de personal capacitado y que dificultan la monitorización en tiempo real, ya que cuando el fenómeno es detectado, su velocidad de propagación ha producido niveles de daño incontrolables.

Una red de sensores inalámbrica o WSN, por sus siglas en inglés, es un sistema distribuido compuesto por nodos con capacidad de obtener información de las condiciones ambientales y transmitirla de manera inalámbrica a una estación base, para su procesamiento [5]. Por otra parte, las técnicas de fusión de información permiten mejorar la calidad de la respuesta ante un evento de interés, al combinar las diferentes fuentes de datos (sensores, base de datos, etc.) [6]. En este artículo, se propone un sistema de detección de incendios forestales en su etapa inicial, utilizando una red de sensores inalámbrica y métodos de fusión de información. La principal contribución de este trabajo es la implementación de un algoritmo de baja complejidad computacional, con la capacidad de detectar un evento de incendio utilizando solo la información de dos sensores: temperatura y humedad.

El resto del documento se encuentra organizado de la siguiente manera. En la Sección II se revisa el trabajo relacionado. Posteriormente, en la Sección III, se describe el método propuesto para la detección de incendios forestales. La Sección IV discute los resultados obtenidos al evaluar el método propuesto. Por último, en la Sección V, se presentan las conclusiones y el trabajo futuro.

\section{Trabajo relacionado}

En la literatura se pueden encontrar diferentes propuestas para la detección de incendios forestales utilizando una WSN. En [7], da Penha et al. proponen dos algoritmos basados en técnicas de fusión de información para la detección de 
incendios utilizando sensores de luz y temperatura. El primero de los algoritmos utiliza el método de umbrales, mientras que el segundo está basado en la teoría de la evidencia de Dempster- Shafer (DST). Las evaluaciones experimentales consistieron en la exposición del nodo sensor ante una antorcha casera utilizada para simular las condiciones de incendio (fuego artificial), a una distancia de 50 a 100 centímetros. El algoritmo basado en DST reportó un número mayor de falsos negativos con respecto al algoritmo basado en umbrales. Sin embargo, los autores resaltan que éste método tiene la ventaja al permitir una extensión rápida de nuevas evidencias (sensores), permitiendo una mayor tasa de detección. En [8], Liu et al. proponen un sistema de detección de incendios utilizando una WSN y una red neuronal artificial. El modelo utiliza cuatro tipos de sensores: temperatura, humedad, luz infrarroja y luz visible. Además, cada nodo sensor es alimentado por una celda solar. El objetivo del sistema es poder realizar detecciones tanto en escenarios diurnos como nocturnos. Sin embargo, reportan dificultades para poder distinguir incendios en estos escenarios. Por tal motivo, se implementó una una red neuronal artificial con multicapa de retropropagación, para fusionar los datos obtenidos por los nodos sensores. Aunque no se presentan detalles de las evaluaciones experimentales, se mencionan cuatro tipos de escenarios: 1) Nodo separado del fuego a $10 \mathrm{~cm}$. 2) Nodo separado del fuego a $20 \mathrm{~cm}$. 3) Nodo separado del fuego a $30 \mathrm{~cm}$. y 4) 'Normal', que representa a un nodo sin presencia del fuego a su alrededor.

Los resultados manifiestan una alta tasa de detección en escenarios de incendio y cero falsas alarmas en escenarios normales. En [9] A. Ko et al. describen un sistema de detección de incendios forestales inteligente. El sistema cuenta con tres subsistemas: cámaras, red de sensores inalámbrica y subsistemas móviles. Estos subsistemas fueron diseñados para verificar los datos obtenidos de forma independiente, y así aumentar la fiabilidad y reducir al mínimo la tasa de falsas alarmas. El subsistema de la cámara combina y analiza imágenes de alta resolución, obtenidas por cámaras infrarrojas de onda corta y cámaras térmicas, para detectar el fuego en el bosque desde varias ubicaciones. La WSN utiliza nodos colocados en lugares estratégicos en todo el bosque, para obtener información sobre la temperatura, humedad, velocidad del viento y otra información local. El subsistema móvil con capacidad de maniobra ofrece datos verificables, que no se pueden obtener a través de los subsistemas estáticos. Sin embargo, el autor no presenta resultados de las evaluaciones.

\section{Modelo de detección propuesto}

Como parte del desarrollo del sistema de detección de incendios, el primer paso fue conocer las características del entorno en condiciones normales (sin presencia de incendio) y en condiciones de incendio. Con el objetivo de desarrollar una propuesta robusta, durante el verano (etapa del año más propensa a los incendios forestales) se colectó información de la temperatura y la humedad relativa en condiciones normales, en una zona arbolada del campus universitario. El análisis de la información arrojó las siguientes observaciones:

1. En condiciones normales, los valores de temperatura y humedad manifiestan un comportamiento cíclico durante las diferentes etapas del día. 
2. En condiciones normales y condiciones de incendio, los valores de temperatura y humedad mantienen una relación inversamente proporcional entre sus magnitudes; es decir, cuando la temperatura se incrementa, la humedad disminuye y viceversa.

3. En condiciones normales, los valores de temperatura y humedad varían muy lentamente durante las diferentes etapas del día. Por otro lado, en condiciones de incendio, la tasa de variación es mayor y se manifiesta en mucho menos tiempo. En la Fig. 1, se observan las mediciones obtenidas de temperatura y humedad en condiciones normales ( $T$ normal y $H$ normal), así como en condiciones de incendio ( $T$ fuego y $H$ fuego), durante el mismo intervalo de tiempo (seis minutos).

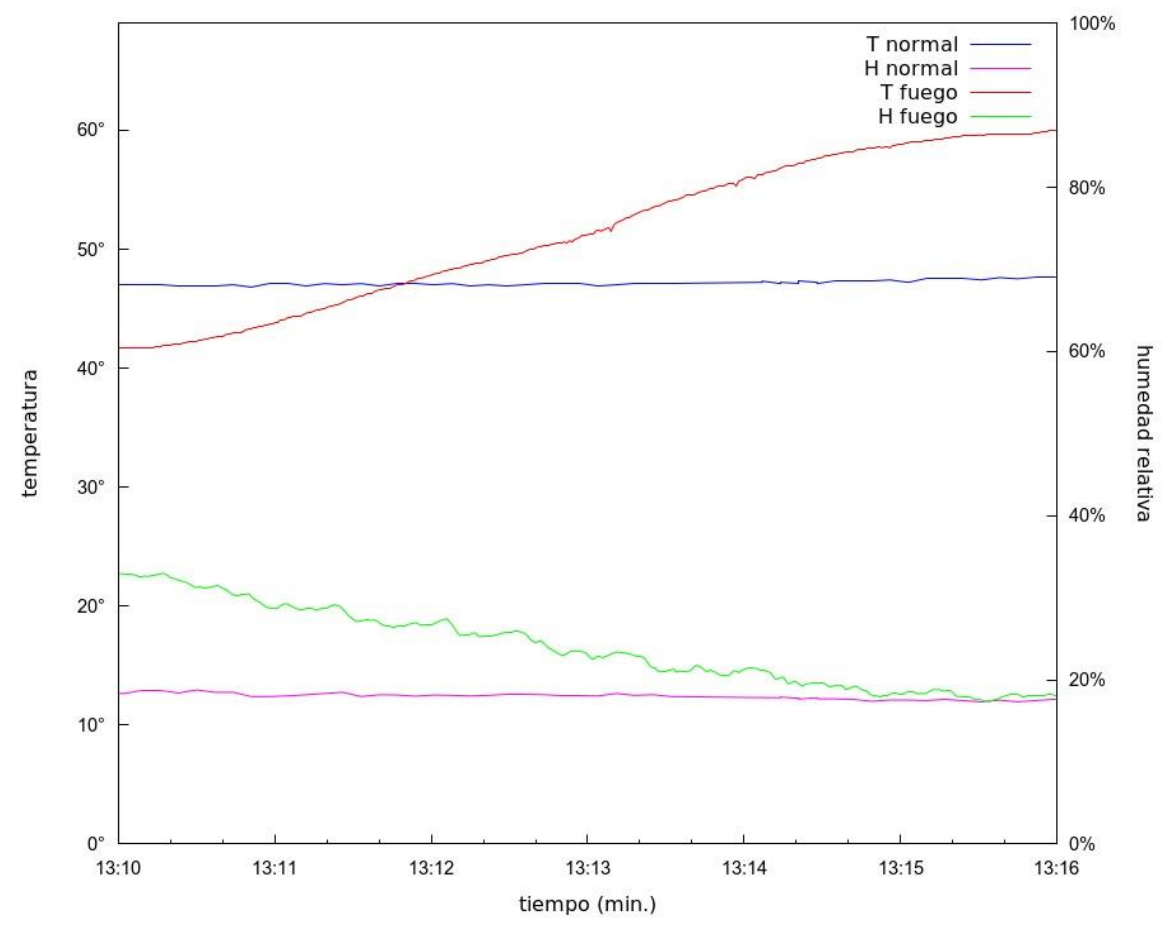

Fig. 1. Valores de temperatura y humedad en condiciones normales e incendio.

4. Cuando un nodo sensor es expuesto a los efectos de los rayos del sol, la temperatura muestra una razón de cambio similar a la producida por un incendio, como se muestra en la Fig. 2.

Tomando en cuenta estas observaciones, se investigaron diferentes técnicas para poder caracterizar el comportamiento de un incendio forestal. En una propuesta inicial, se construyó una función que relacionara el comportamiento de la temperatura y la humedad en un incendio, a la que se nombró función base. Posteriormente, esta función se utilizó para comparar los valores obtenidos por los sensores cuando se registraba un evento que pudiera ser un incendio. A través del uso de técnicas de interpolación y del uso de la teoría de la evidencia de Dempster-Shafer, se determinaba si el evento se trataba de un incendio o no [10]. Sin embargo, a pesar de que se obtuvo una buena tasa de detección, el método no era robusto si las 
condiciones ambientales variaban mucho con respecto a las utilizadas para crear la función base.

En este trabajo, se propone la construcción de la función base utilizando el análisis de regresión. El análisis de regresión ofrece entre sus ventajas la representación de los datos a través del ajuste de una función matemática (línea recta, una función polinomial, una función exponencial, etc.), además de que facilita la estimación de valores desconocidos. En este trabajo, se utilizó el análisis de regresión simple, en el cual existen una variable dependiente y una variable independiente.

Tomando en consideración que los valores de temperatura y humedad relativa mantienen una relación inversamente proporcional en un incendio, se determinó construir dos funciones base, que representen su comportamiento en el incendio, de forma independiente. Del análisis de los parámetros bajo estudio, se encontró que la característica más relevante del incendio es la razón de cambio de los valores de temperatura y humedad con respecto al tiempo. Por tal motivo, al utilizar el análisis de regresión para construir las funciones base, se considera al tiempo como la variable independiente, y a la temperatura y humedad como variables dependientes. Estas funciones son llamadas a partirde ahora como $T(t)$ y $H(t)$, respectivamente, y constituyen el modelo base.

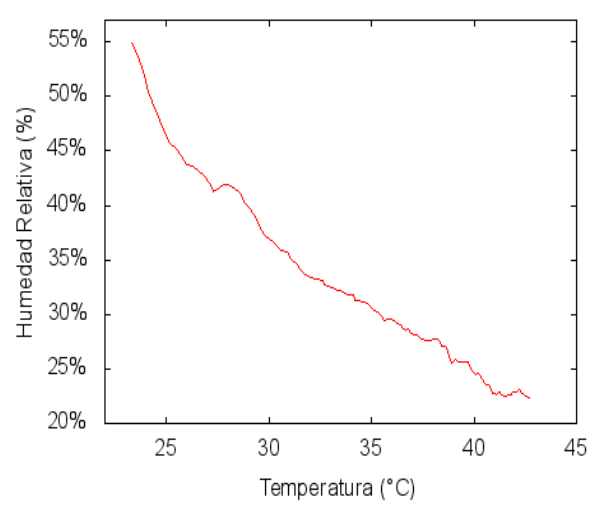

b)

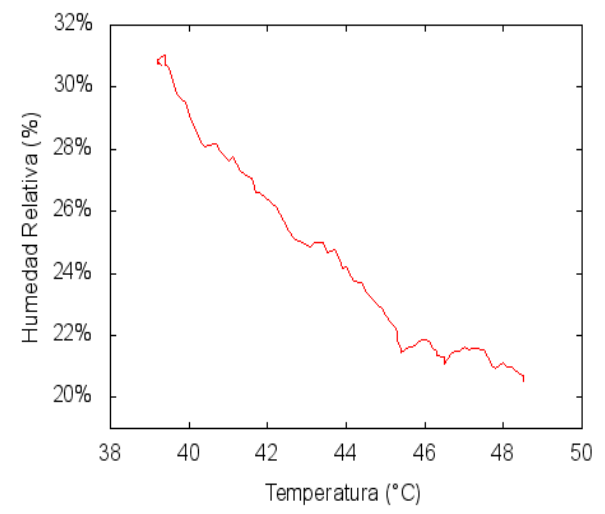

Fig. 2. a) Nodo expuesto a los rayos del sol, b) Nodo expuesto a fuego artificial.

Para la construcción del modelo base, se propone que se utilicen mediciones de temperatura y humedad en experimentos de incendios, considerando alguna época del año y una franja horaria específica. Por ejemplo, pueden construirse modelos base para la temporada de verano, y en diferentes etapas del día (mañana y tarde), que de acuerdo al análisis de datos históricos, fuesen los que han mostrado mayor incidencia de eventos. En la siguiente sección de mostrará un ejemplo de la construcción del modelo base.

El análisis de regresión utiliza el método de mínimos cuadrados para el ajuste de una función a un conjunto de datos. Este método es una manera estandarizada de medir la variación entre los datos y proporciona una función que minimiza la suma de los cuadrados de las diferencias entre la estimación de un modelo de función ideal $\left(\mathrm{y}_{\mathrm{e}}\right)$ y los datos obtenidos por una fuente $\left(\mathrm{y}_{\mathrm{i}}\right)$, como se observa en la Ecuación 14. 


$$
S=\sum_{i=1}^{n}\left(y_{e}-y_{i}\right)^{2}
$$

Por ejemplo, utilizando los datos experimentales que se muestran en la Fig. 3, podemos percibir en los mismos un comportamiento ascendente. El método de mínimos cuadrados permite determinar una función que se ajuste y represente el comportamiento de los datos, como se muestra en la Fig. 3.

La primer opción de función ideal para ajustarse a estos datos es una línea recta; es decir, una ecuación de la forma $\mathrm{y}_{\mathrm{e}}=\mathrm{mx}+\mathrm{b}$. Al sustituir este valor en la Ecuación 1, se obtiene la Ecuación 2.

$$
S=\sum_{i=1}^{n}\left(m x_{i}+b-y_{i}\right)^{2}
$$

Esta nueva ecuación proporciona dos incógnitas: la pendiente $(m)$ y la constante (b). Derivando con respecto a cada una de éstas, se obtienen las Ecuaciones 3 y 4.

$$
\frac{\delta S}{\delta m}=m \sum_{i=1}^{n} x_{i}^{2}+b \sum_{i=1}^{n} x_{i}-\sum_{i=1}^{n} x_{i} y_{i}
$$

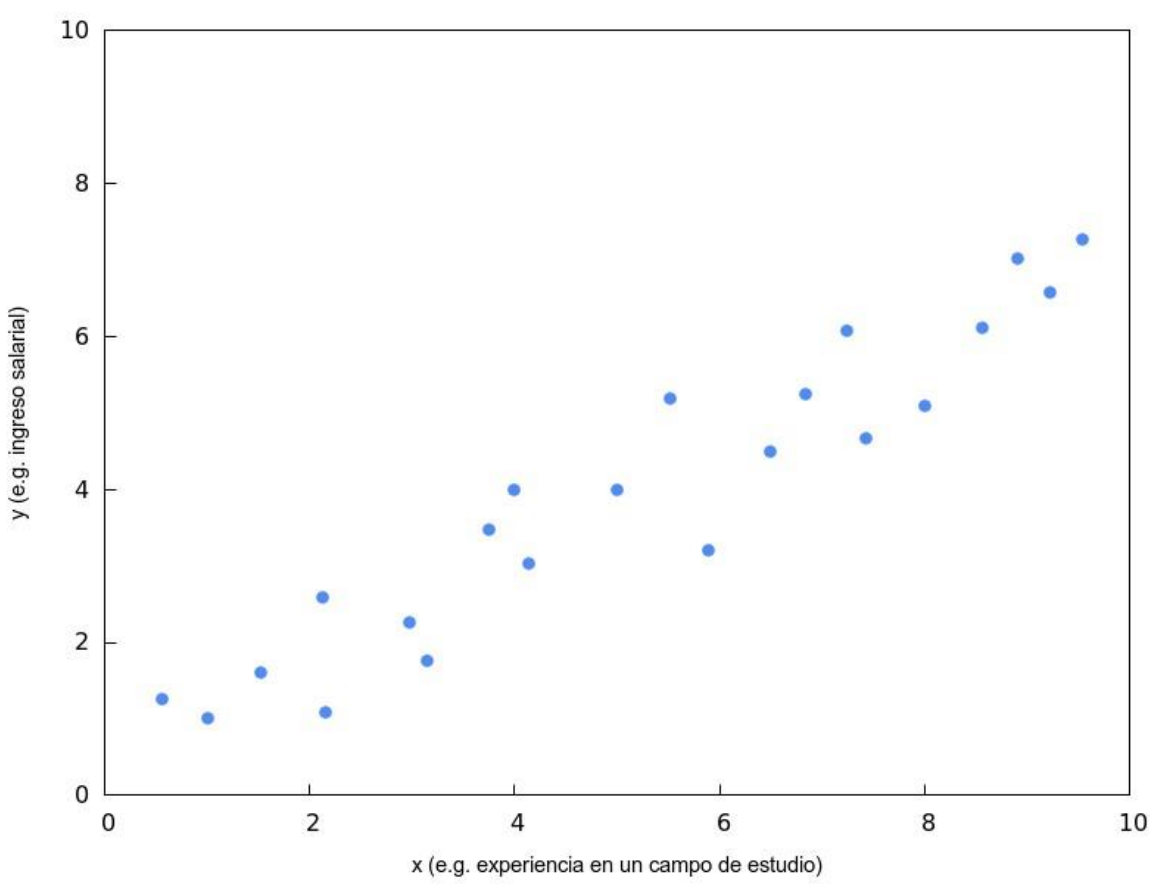

Fig. 3. Ejemplo de datos experimentales.

$$
\frac{\delta S}{\delta b}=m \sum_{i=1}^{n} x_{i}+b n \sum_{i=1}^{n} y_{i}
$$


$\mathrm{Al}$ resolver este sistema de ecuaciones por el método de determinantes, obtenemos las Ecuaciones 5 y 6 :

$$
\begin{gathered}
m=\frac{n \sum_{i=1}^{n} x_{i} y_{i}-\sum_{i=1}^{n} x_{i} \sum_{i=1}^{n} y_{i}}{n \sum_{i=1}^{n} x_{i}^{2}-\left(\sum_{i=1}^{n} x_{i}\right)^{2}} \\
b=\frac{\sum_{i=1}^{n} y_{i}-m \sum_{i=1}^{n} x_{i}}{n}
\end{gathered}
$$

El último paso, consiste en desarrollar y sustituir los resultados de las Ecuaciones 5 y 6 en la Ecuación 2. Esto proporciona una función cuya estimación a los valores dados es lo más cercana posible, como se muestra en la Fig. 4.

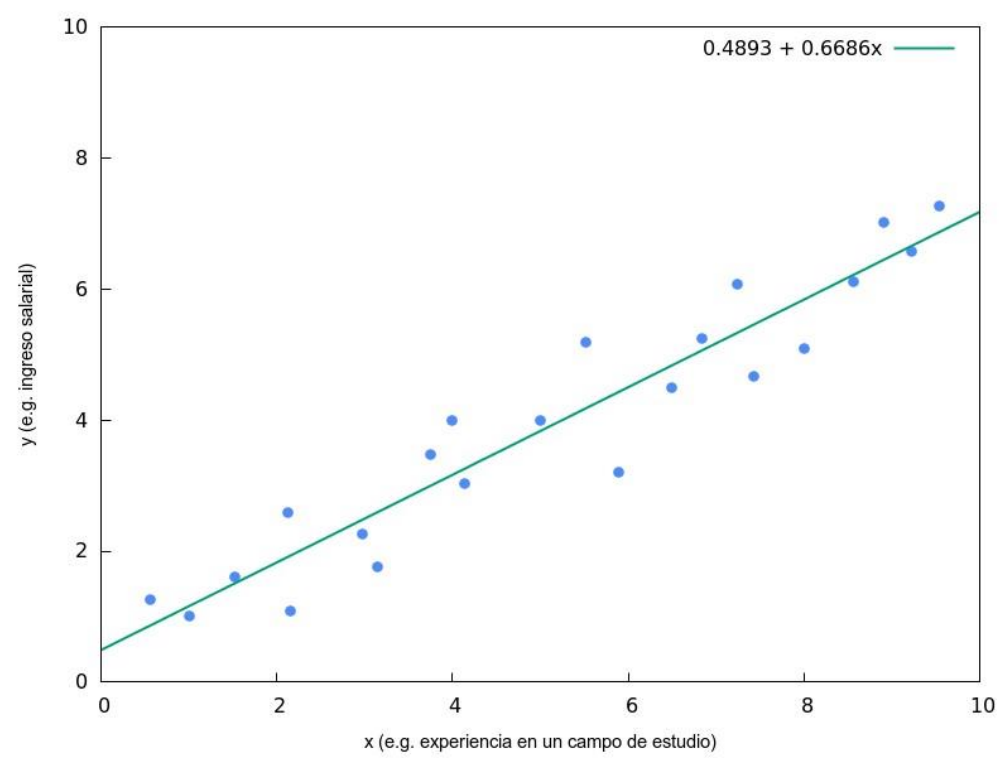

Fig. 4. Función obtenida por el método de mínimos cuadrados.

Sin embargo, la relación entre las variables no siempre se manifiesta de forma lineal como en el ejemplo anterior. Para estos casos existen alternativas como funciones de segundo orden o parábolas, funciones de tercer orden o cúbicas, etcétera. Una manera más general de representar funciones no lineales de orden superior se muestra en la Ecuación 7:

$$
y=\beta_{0}+\beta_{1} x+\beta_{2} x^{2}+\cdots+\beta_{n} x^{n} .
$$

Cabe destacar que también es posible ajustar funciones exponenciales, logarítmicas, entre otras. Por otro lado, uno de los parámetros que validan el resultado del análisis de regresión es la bondad de ajuste. Si los datos se encuentran muy dispersos, la bondad de ajuste será baja; en caso contrario si la dispersión es pequeña entonces la bondad de ajuste será alta. A éste valor se le llama coeficiente de 
determinación o $r^{2}$. El coeficiente de determinación es un valor que oscila entre 0 y 1 , y se calcula de la siguiente manera:

$$
r^{2}=1-\frac{\text { Suma de cuadrados de los residuos }}{\text { Suma de cuadrado total }}
$$

El coeficiente de determinación es un índice que determina la calidad del ajuste de la función obtenida por el análisis de regresión, con respecto una serie de datos en particular. Cuanto más cercano sea $r^{2}$ a 1 , mejor será su calidad de ajuste.

El objetivo del modelo base es contar con una referencia que proporcione mayor precisión al momento de representar las condiciones ambientales existentes en un incendio. Esto permite la posibilidad de no depender de magnitudes fijas o umbrales, sino más bien de la razón de cambio que se produce cuando el fenómeno de incendio afecta al entorno. En otras palabras, de acuerdo con nuestras observaciones durante un incendio forestal, los valores de temperatura y humedad se comportan siempre de la misma manera: ascendente para el caso de la temperatura y descendente para el caso de la humedad relativa, con una razón de cambio o pendiente similar, como puede observarse en la Fig. 5. Contar con un modelo que represente éste comportamiento (independientemente de las magnitudes), permite comparar los datos recibidos por los nodos sensores para afirmar o descartar sí la variación observada corresponde a un incendio. Para tal efecto se propone la siguiente arquitectura, la cual, está compuesta por tres módulos: red de sensores inalámbrica, middleware y sistema de detección de incendios.

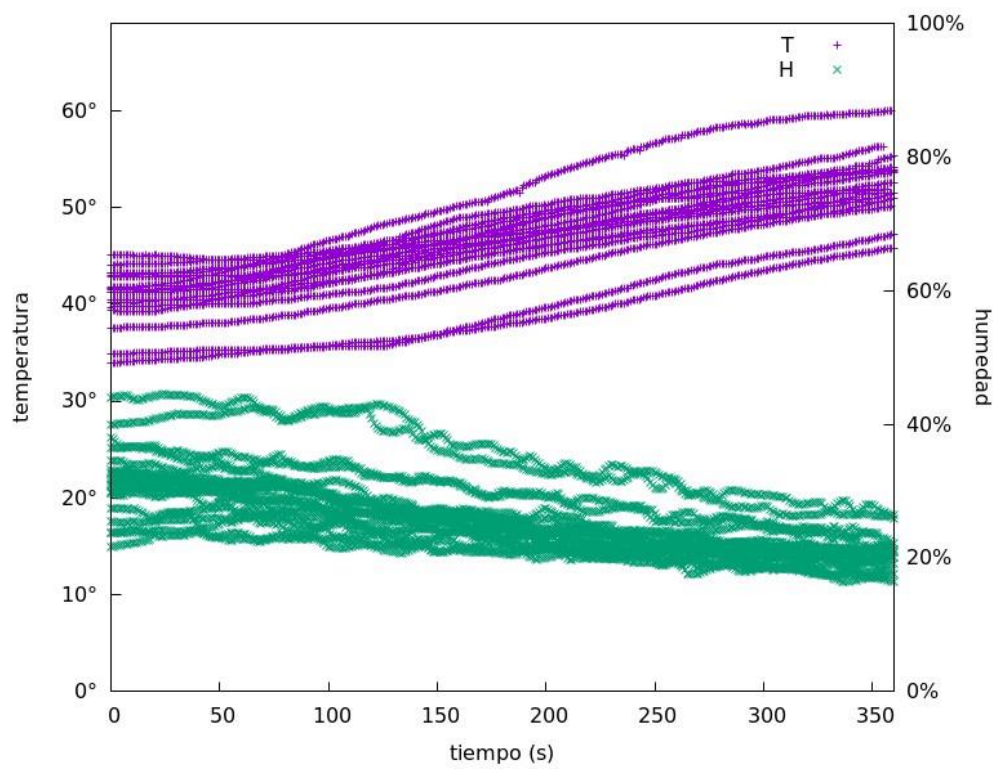

Fig. 5. Valores de temperatura y humedad en un incendio.

En términos generales, el modelo propuesto funciona de la siguiente manera: 1) Los nodos sensores recaban mediciones del entorno periódicamente; 2) cuando detectan un incremento en la temperatura, transmiten la información a la estación 
base; 3) la estación base reenvía los datos al servidor para su almacenamiento en una base de datos; 4) el servidor toma la información más reciente de la base de datos y realiza una comparación entre los datos con respecto a un modelo base previamente definido; y 5) de acuerdo al grado de similitud entre los datos con respecto al modelo base, el sistema determina si existe o no un incendio en la zona monitorizada. A continuación se describen cada una de las etapas de manera mas detallada.

En la red de sensores inalámbrica, cada nodo realiza una lectura de la temperatura utilizando un período de muestreo constante P. Cada lectura es almacenada en una estructura FIFO (First-In, First-Out) llamada $W_{T}$ que utiliza una ventana deslizante de tamaño n. Cuando $\mathrm{W}_{\mathrm{T}}$ se llena, se verifica si la lectura más reciente $T_{n+1}$ ha manifestado un cambio (incremento o decremento), a través de la siguiente razón representada por $\Omega$ :

$$
\Omega=\frac{T_{n+1}}{\mu\left(W_{T}\right)},
$$

donde por otro lado $\mu\left(W_{T}\right)$ equivale al promedio del conjunto de datos sobre la temperatura almacenado en $W_{T}$. El resultado de $\Omega$, será comparado con un umbral de temperatura denominado como $T_{\text {threshold }}$. Sí y solo sí, $\Omega$ es mayor a $T_{\text {threshold }}$, el nodo sensor realiza una lectura de la humedad $H$ y posteriormente transmite un paquete de datos a la estación base con la siguiente información: identificador del nodo id, muestra de temperatura $T$, muestra de humedad $H$ y una estampa de tiempo t para identificar el momento en el que se generó el evento. Para el primer paquete transmitido, el valor de $t$ será igual a cero, y para casos subsecuentes el valor de $t$ estará en función de $P$. En caso no superar el umbral de temperatura, el nodo sensor debe continuar leyendo y evaluando muestras. Éste procedimiento se describe en el Algoritmo 1.

\section{Algoritmo 1 Pre-procesamiento en el nodo sensor}

Sea $T=$ temperatura, $P=$ período de muestreo, $W_{T}=$ buffer de muestras de temperatura, $n=$ tamaño de la ventana deslizante, $\Omega=0, T_{\text {threshold }}=$ umbral de temperatura, $i d=$ identificador del nodo sensor, $H=$ humedad, $t=0, \mathrm{i}=1$;

1. while (1) \{

2. $\quad T=$ Leer la temperatura ambiental;

3. $\quad$ if $(\mathrm{i} \leq n)\{$

4. $\quad W_{T}[\mathrm{i}]=T$;

5. $\quad \mathrm{i}=\mathrm{i}+1$;

6. $\}$ else \{

7. $\Omega=\frac{T}{\mu\left(W_{T}\right)}$

8. $\quad$ while $\left(\Omega>T_{\text {threshold }}\right)\{$

9. $\quad H=$ Leer la humedad ambiental;

10. $\quad$ Enviar $(i d, T, H, t)$;

11. Remover $T$ más antiguo de $W_{T}$;

12. Almacenar $T$ más reciente en $W_{T}$;

13. $t=t+P$;

14. $T=$ Leer la temperatura ambiental; 
15.

16. $\quad$ / /fin del ciclo while

17. $t=0$;

18. $\quad i=1$;

19. $\quad\}$ //fin else

20. \} // Esperar el siguiente período

Cuando el nodo sensor transmite un paquete a la estación base, la información se almacena en una base de datos para su inmediato análisis. La función del middleware es establecer mecanismos de comunicación entre la estación base y el servidor. El middleware asigna un tiempo de arribo compuesto por la fecha y hora del servidor para cada paquete recibido de acuerdo con el identificador del nodo y el equivalente a su respectivo valor $t$.

Posteriormente, el servidor obtiene la información más reciente de la base de datos, tomando en cuenta que cada paquete obtenido por los nodos sensores se compone de los siguientes datos: $i d, T, H$ y $t$. Para describir el flujo del sistema de detección de incendios se utiliza A como el conjunto de información menos reciente o información que rebasó un umbral de temperatura por primera vez y como B la información más reciente o información subsecuente acerca de las condiciones del entorno.

El primer paso es verificar que el tiempo de análisis transcurrido $\Upsilon$, sea menor al tiempo de análisis límite $\Theta$, esto con el objetivo de analizar durante un lapso de tiempo balanceado que permita contar con la información necesaria para tomar una decisión. Al inicio del análisis $\Upsilon=0$, para actualizar su valor debemos acumular el resultado de las diferencias entre $\mathrm{t}_{\mathrm{B}}-\mathrm{t}_{\mathrm{A}}$, como se muestra en la Ecuación 10:

$$
\Upsilon=\Upsilon+\left(t_{B}-t_{A}\right) \text {. }
$$

Suponiendo que una vez recibido un par de muestras (A y B), a su diferencia en el tiempo se le denomina $K$, por lo que $\Upsilon=K$. Para cada nueva muestra se repite el procedimiento mientras el valor de $\Upsilon$ sea menor que $\Theta$ (valor definido por el usuario).

Una vez calculado $\Upsilon$ y validado que es menor a $\Theta$, el siguiente paso es calcular la razón de cambio de los valores de temperatura y humedad de $A$ y $B$ utilizando la fórmula de la pendiente, es decir, $m_{T}$ y $m_{H}$ como se muestra en las Ecuaciones 11 y 12 .

$$
\begin{gathered}
m_{T}=\frac{T_{B}-T_{A}}{t_{B}-t_{A}}, \\
m_{H}=\frac{H_{B}-H_{A}}{t_{B}-t_{A}} .
\end{gathered}
$$

Después debe calcularse la derivada de las funciones que representan el modelo base, es decir, $T(t)$ y $H(t)$. Sin embargo, cabe mencionar que $T^{\prime}(t)$ y $H^{\prime}(t)$ deben obtener un resultado equivalente al valor de $\Upsilon$, por lo cual, éstas se sustituyen por $T^{\prime}(\Upsilon)$ y $H^{\prime}(\Upsilon)$ Posteriormente se utiliza la técnica del error cuadrático promedio utilizando un número de evaluaciones límite denominado como emax. El error cuadrático promedio es utilizado para medir el error entre un estimador (funciones del 
modelo base) y una estimación (datos provenientes del nodo sensor). Este valor se calcula como se muestra en las Ecuaciones 13 y 14:

$$
\begin{aligned}
M S E_{T} & =\frac{\sum_{i=0}^{e \max }\left(T_{i}^{\prime}(\Upsilon)-m_{T_{i}}\right)^{2}}{e \max }, \\
M S E_{H} & =\frac{\sum_{i=0}^{e \max }\left(H_{i}^{\prime}(\Upsilon)-m_{H_{i}}\right)^{2}}{e \max } .
\end{aligned}
$$

Con el objetivo de clasificar los resultados del error cuadrático promedio, se definió una tabla que proporciona un valor de masa con dominio entre 0 y 1 , donde cero representa un alto grado de similitud (entre 90 y 100 por ciento), mientras que 1 representa un grado casi nulo de similitud con respecto a los datos evaluados. El valor de masa es utilizado como resumen de la serie de comparaciones del MSE. El último

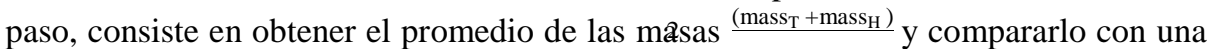
constante $\alpha$. Este último valor es utilizado para determinar si el sistema debe o no, enviar una alerta de incendio al usuario.

\section{Evaluación}

Con la finalidad de evaluar el desempeño del modelo propuesto, se llevaron a cabo una serie de experimentos simulando incendios forestales. En esta sección se presentan los resultados obtenidos.

Para llevar a cabo el conjunto de experimentos de incendios fueron utilizados nodos sensores con plataforma de hardware IRIS, con una placa sensora modelo MTS420/400CC [11]. Esta placa tiene integrados cinco tipos de sensores ambientales: humedad relativa, temperatura, presión barométrica, intensidad luminosa y acelerómetro. En cuestiones de software, en el servidor se utilizó Ubuntu Linux versión 12.04. El middleware fue desarrollado en Java y la base de datos en MySQL. Por último, para la WSN se utilizó el sistema operativo TinyOS en su versión 2.1.2.

La metodología utilizada consistió en colocar el nodo sensor sobre la parte media del tronco de un árbol, a una altura aproximadamente de tres metros. Los experimentos se llevaron a cabo durante los meses de Abril y Mayo de 2015. Por otra parte, la recolección de datos fue dividida en dos etapas: 1) Creación de modelo base y 2) Experimentos de fuego controlado. A continuación se describe cada una de ellas.

Tabla 1. Parámetros utilizados en experimentos

\begin{tabular}{ccc}
\hline Parámetro & Descripción & Valor \\
\hline $\mathrm{n}$ & Tamaño de la ventana $\mathrm{W}_{\mathrm{T}}$ & 15 \\
$\mathrm{~T}_{\text {threshold }}$ & Umbral de temperatura & 1.01 \\
$\mathrm{P}$ & Período de muestreo del nodo sensor & 6 segundos \\
$\Theta$ & Tiempo de análisis límite & 360 segundos \\
emax & No. de evaluaciones máximo para el MSE & 5 \\
$\alpha$ & Umbral utilizado para determinar un incendio & 0.7 \\
\hline
\end{tabular}

1. Modelo base: Con el objetivo de contar con suficiente información para el desar- rollo de las funciones base $T(t)$ y $H(t)$, en esta etapa el nodo sensor fue 
expuesto a condiciones de fuego artificial utilizando una antorcha para jardín. La duración del experimento fue de 6 minutos utilizando un período de muestreo de 1 segundo. En esta etapa se realizaron 20 experimentos.

2. Experimentos de fuego controlado: Se llevaron a cabo series de experimentos con diferentes períodos de muestreo: 3, 4, 6 y 8 segundos. El objetivo fue investigar el período de muestreo con mejor desempeño para la detección del evento. En esta etapa se realizaron 32 experimentos ( 8 por cada tipo de período de muestreo utilizado). La finalidad fue determinar el período de muestreo a utilizar en la siguiente etapa de experimentos.

Para construir el nuevo modelo base, se implementó el ajuste de curva por análisis de regresión simple con la ayuda del software de análisis estadístico SPSS de IBM

[12]. Con los datos recabados, se obtuvo el siguiente modelo base:

$$
\begin{gathered}
\mathrm{T}(\mathrm{t})=-2.477 \times 10^{-7} \mathrm{t}^{3}+1.12 \times 10^{-4} \mathrm{t}^{2}+2.2441 \times 10^{-2} \mathrm{t}+28.8861, \\
\mathrm{H}(\mathrm{t})=2.26 \times 10^{-8} \mathrm{t}^{3}-1.7 \times 10^{-5} \mathrm{t}^{2}-2.7444 \times 10^{-2} \mathrm{t}+17.505
\end{gathered}
$$

Cabe mencionar que los coeficientes de determinación fueron de 0.87 para la función $T(t)$ y 0.68 para la función $H(t)$.

Posteriormente, se llevaron a cabo 50 experimentos de incendio, utilizando los parámetros que se muestran en la Tabla 1, 30 de ellos escenarios de sombra y con nodo sensor expuesto a fuego artificial. Los 20 restantes fueron eventos sin incendio y con el nodo sensor fue expuesto a los rayos del sol, sin protección alguna. Estos valores fueron procesados utilizando el modelo base descrito por las Ecuaciones $15 \mathrm{y}$ 16. El modelo obtuvo una tasa de detección del $100 \%$ en escenarios de sombra. Al realizar el análisis de los datos obtenidos por un nodo sensor expuesto a los rayos del sol, se obtuvo una tasa de $100 \%$ de falsos positivos. Sin embargo para evitar esto, se puede cubrir al nodo sensor con protección especial para evitar la exposición directa a la luz solar. Para comprobarlo, se llevaron a cabo un nuevo conjunto de 50 experimentos con el nodo sensor expuesto a los rayos del sol, pero cubriendo a éste con una protección. Al utilizar el método propuesto en estas condiciones, se obtuvo una tasa de detección del $100 \%$.

\section{Conclusiones y trabajo futuro}

En este trabajo se propuso un sistema de detección de incendios forestales en su etapa inicial utilizando una red de sensores inalámbrica y métodos de fusión de información. Su principal característica es que utiliza tan solo información de los sensores de temperatura y humedad. Su baja complejidad computacional lo hace ideal para monitorizar zonas de riesgo y emitir alarmas en tiempo real. El modelo requiere del desarrollo modelo base que represente las características de las condiciones ambientales existentes en un incendio. El modelo base se construye utilizando análisis de regresión. Cuando el sistema detecta valores de temperatura y humedad que pudieran representar un incendio, compara los valores recabados con el modelo base para determinar si existe o no un incendio. Los resultados de la evaluación del modelo mostraron una tasa de detección del $100 \%$ cuando los nodos no están expuestos directamente a los rayos del sol. Como trabajo futuro, se busca extender el modelo 
considerando el consumo de energía de la WSN, a través de la distribución de los nodos en clusters y el uso de sensado distribuido.

\section{Referencias}

1. Ali, A.A., Carcaillet, C., Gauthier, S., Hély, C., Le Goff, H., Terrier, A., Bergeron Yves, G., Martin, P.: Fire in managed forests of eastern Canada: Risks and options. Forest Ecology and Management, 294, pp. 238-249 (2013)

2. CONAFOR. Incendios forestales en México temporada, http://www.conafor.gob. $\mathrm{mx} / \mathrm{web} /$ temas-forestales/incendios/ (2014)

3. AVHRR. http://noaasis.noaa.gov/NOAASIS (2015)

4. MODIS. http://modis.gsfc.nasa.gov/ (2015)

5. Yick, J., Mukherjee, B., Ghosal, D.: Wireless sensor network survey, Computer Networks, 52(12), pp. 2292-2330 (2008)

6. Nakamura, E.F., Loureiro, A.F., Frery, A.C.: Information fusion for wireless sensor networks: Methods, models, and classification, ACM Comput. Surv., 39(3) (September 2007)

7. da Penha O.S., Nakamura, E.F.: Fusing light and temperature data for fire detection. In: Computers and Communications (ISCC), IEEE Symposium on, pp. 107-112 (June 2010)

8. Liu, Y., Gu, Y., Chen, G., Ji, Y., Li, J.: A novel accurate forest fire detection system using wireless sensor networks. In: Mobile Ad-hoc and Sensor Networks (MSN), 2011 Seventh International Conference on, pp.52-59 (Dec 2011)

9. Ko, A., Lee, N.M.Y., Sham, R.P.S., So, C.M., Kwok, S.C.F.: Intelligent wireless sensor Network for wildfire detection. 158, pp. 137-148 (2012)

10. Díaz-Ramírez, A., Tafoya, L.A., Atempa, J.A., Mejía-Alvarez, P.: Wireless sensor networks and fusion information methods for forest fire detection, Procedia Technology, 3(0), pp.69-79. In: The 2012 Iberoamerican Conference on Electronics Engi- neering and Computer Science (2012)

11. MEMSIC. Product datasheet. http://www.memsic.com/ (Enero 2014)

12. IBM. http://www-01.ibm.com/software/analytics/spss/ (2014) 